\title{
ALGAS MARINAS DE ISLA VERDE, VERACRUZ, MEXICO
}

\author{
Luz Elena Mateo-Cid \\ A. Catalina Mendoza-Gonzalez ${ }^{1}$ \\ Y \\ Citlalli Galicia Garcia \\ Departamento de Botánica \\ Escuela Nacional de Ciencias Biológicas, I.P.N. \\ Carpio y Plan de Ayala \\ México, D.F. 11340
}

\section{RESUMEN}

Se presentan los resultados obtenidos del estudio de las algas bentónicas de Isla Verde, Veracruz, en aguas del Golfo de México. La lista florística se acompaña de datos sobre la estacionalidad de las especies y su estadio reproductivo, el nivel de marea, el sustrato, la exposición al oleaje y el epifitismo asociado a ellas.

Se determinaron 5 especies de Cyanophyceae, 37 de Rhodophyceae, 6 de Phaeophyceae, 8 de Bacillariophyceae y 24 de Chlorophyceae. La flora encontrada en Isla Verde es tropical y típica de los arrecifes coralinos. Se discute la diversidad de las algas con respecto a sustrato, luz y temperatura.

\section{ABSTRACT}

A study on benthic marine algae from Isla Verde, Veracruz in the waters of the Gulf of Mexico revealed the existence of 5 species of Cyanophyceae, 37 species of Rhodophyceae, 6 of Phaeophyceae, 8 species of Bacillariophyceae and 24 of Chlorophyceae. The study includes information on seasonality, reproductive stage, tidal level, wave exposure, substrate and epiphytism. The species composition found in Isla Verde is of tropical affinity and representative of the algal flora associated with coral reefs as was expected. Species diversity in relation to substrate, ligth and temperature is discussed.

\section{INTRODUCCION}

Los arrecifes coralinos se encuentran entre los ecosistemas más ricos de nuestro planeta; junto con los manglares, lagunas costeras y estuarios forman ambientes de alta productividad. La distribución de los arrecifes de coral se encuentra limitada a las provincias Indopacífica y del Caribe, entre los $35^{\circ} \mathrm{N}$ y los $32^{\circ} \mathrm{S}$. Prosperan en condiciones ambientales específicas como son: temperaturas que oscilan entre 22 y $28^{\circ} \mathrm{C}$, aguas transparentes y salinidad de 34 a 37 ppm. (Lot-Helgueras, 1971).

\footnotetext{
${ }^{1}$ Profesora becaria de la Comisión de Operación y Fomento de Actividades Académicas del I.P.N.
} 
Las costas veracruzanas poseen complejos arrecifales de alta relevancia ecológica; la principal característica física común entre ellos es su posición y forma que se alarga en sentido NW a SE en función de la dirección de las corrientes (Lot-Helgueras, 1971).

Los estudios sobre la flora ficológica bentónica de los arrecifes coralinos veracruzanos no son abundantes, los trabajos ficoflorísticos más importantes que se han llevado a cabo en esta región son los de Humm y Hildenbrand (1962) y Emery (1963), quienes describen la presencia de ciertas algas en los arrecifes Lobos y Enmedio; Huerta y Garza (1964) así como Rigby y Mclntire (1966), citan las algas que dominan en Tuxpan, La Blanquilla y Lobos. Villalobos (1971) realiza un estudio sobre las algas marinas del arrecife Blanquilla; Chávez (1973), en su estudio del arrecife Lobos, indica cuales son las algas dominantes y de fácil observación en ese ecosistema. Huerta et al. (1977) presentan una lista sistemática de las algas marinas de Isla de Enmedio. Henkel (1982) describe la presencia de algunas algas en los Arrecifes Lobos y Enmedio. Mendoza-González y Mateo-Cid (1985) registran la flora marina de las islas Sacrificios y Santiaguillo; recientemente Tunnell (1988) y Lehman y Tunnell (1992) describen la composición y ecología de las macroalgas de Isla de Enmedio.

Por lo que respecta a Isla Verde, ésta ha sido estudiada por Huerta (1960), LotHelgueras (1968) y Quintana y Molina (1991); en tales trabajos se citan 32 especies de algas marinas para el arrecife de este cuerpo insular.

\section{ZONA DE ESTUDIO}

Isla Verde se encuentra localizada dentro del sistema arrecifal veracruzano, en las coordenadas 9604'06" de longitud oeste y los 19¹1'50" de latitud norte (Fig. 1). Está ubicada a $5.37 \mathrm{~km}$ del puerto de Veracruz, tiene una extensión de $1214 \mathrm{~m}$ de longitud y $750 \mathrm{~m}$ en su parte más ancha; presenta una porción emergida en el sur del arrecife con $300 \mathrm{~m}$ de largo y $170 \mathrm{~m}$ de ancho (Lot-Helgueras, 1971).

La zona de barlovento presenta sustrato constituido por roca basáltica; la barrera coralina que encierra a la laguna central se interrumpe en la porción este constituyendo una boca de $50 \mathrm{~m}$, frente a la cual se localiza una fosa de $10 \mathrm{~m}$ de profundidad. El sustrato del arrecife está formado principalmente por sedimentos calcáreos provenientes de los corales madreporianos. Las conchas de moluscos, las algas rojas calcificadas y las especies del género Halimeda son contribuyentes secundarios (Emery, 1963).

De acuerdo con el sistema climático de Köppen, modificado por García (1973), el área de estudio tiene un clima del tipo $\mathrm{A}\left(\mathrm{w}_{2} "\right)(\mathrm{w})\left(\mathrm{i}^{\prime}\right)$, caliente húmedo con lluvias en verano; existen dos estaciones climáticas bien definidas; la de secas o época de "nortes" que abarca de septiembre hasta abril, con escasa precipitación, temperatura baja y frecuentes invasiones de masas de aire frío del norte y, la de lluvias, que corresponde a los meses de mayo a agosto, que es un período cálido, caracterizado por temperatura elevada y alta precipitación. El litoral de Veracruz se encuentra influenciado por la corriente del Golfo de México, cuya circulación está vinculada con las aguas cálidas y salinas que constituyen 
Mateo-Cid et al.: Algas Marinas de Isla Verde, Veracruz, México

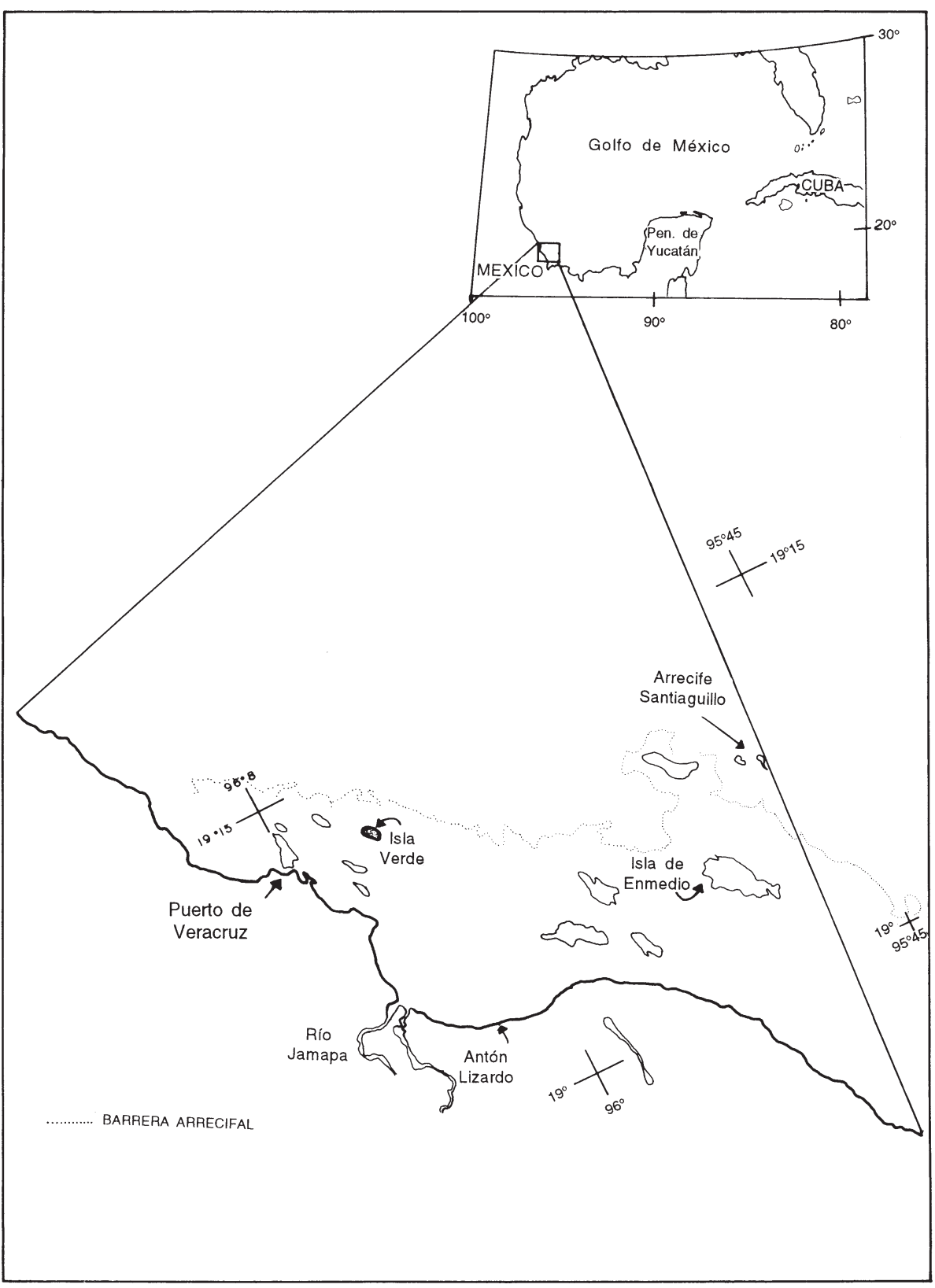

Fig. 1. Localización de la zona de estudio. 
la Corriente de Lazo; esta corriente tiene salinidad de $36.7 \mathrm{ppm}$ y temperatura superficial durante el verano de 28 a $29^{\circ} \mathrm{C}$, la que se reduce de 25 a $26^{\circ} \mathrm{C}$ en el invierno (Anónimo, 1982; de la Lanza, 1991; Vargas-Hernández et al., 1993). La marea es de tipo mixto, la pleamar superior es de 0.84 m y la bajamar de -0.24 m (Lot-Helgueras, 1971; Anónimo, 1982).

\section{METODOLOGIA}

Las muestras biológicas se obtuvieron durante 1983, 1984, 1985, 1986 y 1992 mediante seis visitas, una en la época de lluvias y cinco en la estación climática de secas.

El material ficológico fue recolectado a mano en el nivel intermareal con ayuda de espátulas y navajas de campo; en el nivel submareal se obtuvo por medio de buceo libre entre $0.5-2.0 \mathrm{~m}$ de profundidad.

Las muestras se fijaron en una solución de formaldehido a $4 \%$ en agua de mar. El material fue procesado y depositado en el herbario ENCB. El número de registro de herbario de los ejemplares correspondientes se encuentra indicado en la columna de observaciones (OBS) del Cuadro 1. Para la descalcificación de las algas se usó $\mathrm{HCl}$ 1:2. La determinación del material ficológico se llevó a cabo utilizando los trabajos de Taylor (1960), Werner (1977), Humm y Wicks (1980), Norris (1987), Anagnostidis y Komárek (1988), Huisman y Borowitzka (1990), Penrose y Woelkerling (1991), Schneider y Searles (1991) y Penrose y Chamberlain (1993). La secuencia de la lista florística va acorde con las obras de Werner (1977), para Bacillariophyceae, con la de Wynne (1986) para Rhodophyceae, Phaeophyceae y Chlorophyceae y con la de Komárek y Anagnostidis (1986) para Cyanophyceae.

Para cada especie se presentan datos referentes a su estacionalidad en el área de estudio y su estadio reproductivo, la exposición al oleaje, el sustrato, el nivel de marea en el que se desarrollan y el epifitismo.

\section{RESULTADOS Y DISCUSION}

Flora

Para Isla Verde se determinaron 80 especies de algas marinas bentónicas; de este total, 5 (6.25 \%) corresponden a Cyanophyceae, 37 (46.25\%) a Rhodophyceae, 6 (7.5 \%) a Phaeophyceae, 8 (10\%) a Bacillariophyceae y 24 (30\%) a Chlorophyceae.

En la estación climática de lluvias se localizaron 28 taxa y en la de secas 79.

Las familias Corallinaceae y Rhodomelaceae fueron las mejor representadas en cuanto al número de especies se refiere, con 14 y 7 respectivamente.

Considerando que la temperatura media anual superficial del agua en las costas de Veracruz es de $26^{\circ} \mathrm{C}$ (Lot-Helgueras, 1971), la ficoflora aquí listada queda comprendida dentro del intervalo tropical (Dawes, 1986). 
Principales comunidades y su habitat

La distribución de las algas marinas está determinada principalmente por la temperatura y la luz. Otros factores tales como el tipo de sustrato y las mareas determinan asimismo la composición florística y abundancia de cada comunidad (Dawes, 1986).

Los arrecifes coralinos están bien representados en aquellos lugares de las plataformas insulares y continentales donde las características físicas y ecológicas les son favorables. En la parte más expuesta del arrecife se desarrollan masas de rodofíceas incrustantes; pero entre las comunidades de madréporas el predominio es animal y el habitat resulta menos propicio para el establecimiento de las algas bentónicas (DíazPiferrer, 1967).

El establecimiento de algunas especies de algas bentónicas en los arrecifes se relaciona con la zonación o estratificación de las asociaciones o comunidades de corales vivos; así, en los niveles superiores, aparecen creciendo a la sombra de Porites especies como Halimeda opuntia (Linnaeus) Lamouroux, H. tuna (Ellis et Solander) Lamouroux y Caulerpa cupressoides (Vahl) C. Agardh.

En la plataforma arrecifal de Isla Verde se distinguen varias comunidades: en la porción oeste existe una clara zonación, a partir de la línea de costa hacia la plataforma se localiza una fitocenosis dominada por Acetabularia shenckii Möbius, Polyphysa polyphysoides (P. et H. Crouan) Schnetter, Neomeris annulata Dickie, Cladophora socialis Kützing, Boodlea composita (Harvey) Brand, Dictyosphaeria cavernosa (Försskal) Boergesen y Ernodesmis verticillata (Kützing) Boergesen, las que se desarrollan en fragmentos de coral antiguo y piedra caliza. Esta comunidad se ve afectada por los cambios en el nivel del agua provocados por el ritmo mareal. Round (1981) menciona que las Chlorophyta sifonadas son las que están mejor representadas en la franja interna de la planicie arrecifal; esta zona, conocida como intermareal, es habitada por plantas que son capaces de tolerar factores ambientales rigurosos, tales como una fluctuación drástica de la temperatura y de la salinidad, desecación periódica, alta intensidad lumínica y exposición al viento y a la lluvia (Dawes, 1986).

Enseguida se encuentra la comunidad de Thalassia testudinum König, siempre protegida, de sustrato arenoso y caracterizada por la presencia de algas verdes psamófilas de los géneros Rhipocephalus, Halimeda y Caulerpa; mientras que sobre los restos de coral se ubican Galaxaura rugosa (Ellis et Solander) Lamouroux, Liagora ceranoides Lamouroux, Laurencia obtusa (Hudson) Lamouroux, Digenea simplex (Wulfen) C. Agardh y Acanthophora spicifera (Vahl) Boergesen; allí mismo se encuentran las numerosas epífitas de Thalassia, principalmente las algas rojas incrustantes Hydrolithon farinosum (Lamouroux) Penrose y Chamberlain y Pneophyllum fragile Kützing, así como Cladosiphon zosterae (J. Agardh) Kylin, Calothrix crustacea Thuret, Champia parvula (C. Agardh) Harvey, Spyridia filamentosa (Wulfen) Harvey y Chondria curvilineata Collins et Hervey, entre otras. El mayor número de especies encontradas en Isla Verde se localizó en la mencionada comunidad. Cabe hacer notar que el género Halimeda se observó de modo abundante aquí; Lüning (1990) señala que esta alga calcificada es un importante constructor del arrecife ya que deposita $\mathrm{CaCO}_{3}$, en forma de aragonita, en la superficie de sus talos y éstos con el tiempo contribuyen a incrementar el sustrato; tanto Halimeda como Rhipocephalus pueden ser considerados como significativos edificadores algales de este ecosistema. 
Después de la comunidad de Thalassia, encontramos la plataforma arrecifal, la cual tiene una superficie irregular y una profundidad máxima de $2 \mathrm{~m}$; el sustrato está formado por coral antiguo, piedra caliza y arena. En esta zona se registran numerosos representantes del orden Corallinales, principalmente de formas incrustantes como Hydrolithon improcerum (Foslie) Foslie et Howe, Lithophyllum bermudense Foslie et Howe, L. congestum (Foslie) Foslie, Neogoniolithon spectabile (Foslie) Setchell et Mason y Paragoniolithon solubile (Foslie et Howe) Adey, Townsend et Boykins; además de Galaxaura rugosa, Amphiroa rigida var. antillana Boergesen y otras especies de algas cafés como Padina boergesenii Allender et Kraft y Dictyota cervicornis Kützing. En la comunidad dominan las coralinas incrustantes las que se localizaron durante todo el periodo de estudio, siendo las algas filamentosas y laminares las menos frecuentes. Este comportamiento coincide con lo encontrado por Littler et al. (1983) para el arrecife Carrie Bow en Belize, quienes observaron que las especies calcáreas costrosas y articuladas son más resistentes al pastoreo por peces que las especies de algas filamentosas y laminares.

Los géneros tales como Paragoniolithon, Lithophyllum y Neogoniolithon contribuyen al incremento vertical del arrecife y forman barreras que pueden 0 no estar expuestas. En los arrecifes coralinos alrededor de $20 \%$ de las especies de peces son herbívoras y las algas coralinas costrosas son beneficiadas por el pastoreo de estos animales (Lüning, 1990). En los estudios realizados por Littler y Doty se demostró que gracias al pastoreo de los herbívoros la luz puede alcanzar la superficie del arrecife, permitiendo así el crecimiento de Porolithon en las aguas profundas de la pendiente (Round, 1981).

Por último ubicamos el borde coralino del lado noreste-este, el cual sobresale en forma aparente por el denso crecimiento de coral vivo. La zona se encuentra en barlovento y en este borde o barrera arrecifal, que recibe el embate directo de las olas, sólo se localizaron algunas especies coralinas incrustantes y Padina boergesenii, ya que la acción de las olas remueve a los competidores y tal efecto permite que las algas rojas costrosas prosperen en este lugar (Round, 1981).

\section{Especies Epífitas}

Muchas algas marinas crecen como epífitas en otras plantas (algas, pastos marinos y raíces de mangle). En algunos casos, la presencia de la epífita en un hospedante puede ser fortuita, ya que ésta se comporta como especie oportunista y se sirve de una gran variedad de hospedantes. Otras veces existe un alto grado de especificidad y tales algas se presentan sólo en un número limitado de hospedantes, siendo por ello epífitas estrictas u obligadas (Goff y Coleman, 1985; Ibarra-Obando y Aguilar-Rosas, 1985).

En este estudio se determinaron 29 especies epífitas, 12 de las cuales se observaron exclusivamente sobre Thalassia, cuatro más también se encontraron como habitantes estrictos de otras algas y las 13 restantes se registraron sin preferencia por algún hospedero.

De las 29 epífitas, 13 corresponden a Rhodophyceae, 8 a Bacillariophyceae, 5 a Cyanophyceae y 3 a Phaeophyceae. El número más alto de ellas se localizó en la época de secas, con 29 especies, lo que puede deberse a que las poblaciones de macroalgas se encontraban en decadencia y por lo tanto eran más sensibles a ser colonizadas; por otro lado las condiciones de deterioro en la superficie de las hojas del pasto marino favorecían el establecimiento de las epífitas. 
Reproducción

El tipo de reproducción que predominó en las especies enumeradas en este estudio, principalmente de Rhodophyceae, fue el asexual, que tiene las ventajas de requerir de un menor gasto de energía para la formación de las esporas y diseminar éstas en forma rápida, lo que permite una dispersión eficiente de las mismas (Santelices, 1977). Las Phaeophyceae se observaron en reproducción sexual. En cuanto a Chlorophyceae, un gran número de sus representantes se localizó en estado vegetativo, ya que las fases sexuales son poco notorias y efímeras (Fritsch, 1935).

\section{AGRADECIMIENTOS}

A la Secretaría de Marina por el apoyo proporcionado para la realización del trabajo de campo. Carlos Barbosa, Magnolia Nava y Bertha López colaboraron en la recolección del material biológico. Ma. Guadalupe Tejeda Hernández capturó la información y Dante Espinoza Cid elaboró la figura.

Cuadro 1. Algas marinas de Isla Verde, Veracruz.

\begin{tabular}{|c|c|c|c|c|c|c|c|}
\hline Especies & Presencia & \begin{tabular}{|} 
Repro- \\
ducción
\end{tabular} & $\begin{array}{c}\text { Nivel } \\
\text { marea }\end{array}$ & $\begin{array}{l}\text { Exp. al } \\
\text { oleaje }\end{array}$ & Sustrato & $\begin{array}{c}\text { Epífita } \\
\text { de }\end{array}$ & $\begin{array}{c}\text { Observa- } \\
\text { ciones }\end{array}$ \\
\hline $\begin{array}{l}\text { CYANOPHYCEAE } \\
\text { Chamaesiphonaceae } \\
\text { 1. Entophysalis conferta } \\
\text { (Kützing) Drouet et Daily }\end{array}$ & $S$ & & L & Ex & & 52 & $\mathrm{Ee}$ \\
\hline $\begin{array}{l}\text { Chroococcaceae } \\
\text { 2. Anacystis aeruginosa } \\
\text { (Zanardini) Drouet et Daily }\end{array}$ & $S$ & & I & $\begin{array}{l}P \\
\text { Ex }\end{array}$ & & $\begin{array}{l}10 \\
33 \\
52 \\
78\end{array}$ & $E$ \\
\hline $\begin{array}{l}\text { Nostocaceae } \\
\text { 3. Calothrix crustacea Thuret }\end{array}$ & $S$ & $\mathrm{Ht}$ & 1 & $P$ & & $\begin{array}{c}\mathrm{S} / \mathrm{Th} \\
78\end{array}$ & $E$ \\
\hline $\begin{array}{l}\text { Oscillatoriaceae } \\
\text { 4. Blennothrix lyngbyacea } \\
\text { (Kützing) Anagnostidis } \\
\text { et Komárek }\end{array}$ & $S$ & $\mathrm{Hm}$ & $\begin{array}{l}\text { I } \\
\text { L }\end{array}$ & $\begin{array}{l}P \\
\text { Ex }\end{array}$ & & $\begin{array}{l}10 \\
33 \\
52 \\
74\end{array}$ & $\begin{array}{c}10686 \\
7902 \\
E\end{array}$ \\
\hline
\end{tabular}


Acta Botánica Mexicana (1996), 36:59-75

Cuadro 1. Continuación.

\begin{tabular}{|c|c|c|c|c|c|c|c|}
\hline Especies & Presencia & $\begin{array}{l}\text { Repro- } \\
\text { ducción }\end{array}$ & $\begin{array}{c}\text { Nivel } \\
\text { marea }\end{array}$ & \begin{tabular}{|l} 
Exp. al \\
oleaje
\end{tabular} & Sustrato & $\begin{array}{c}\text { Epífita } \\
\text { de }\end{array}$ & $\begin{array}{l}\text { Observa- } \\
\text { ciones }\end{array}$ \\
\hline $\begin{array}{l}\text { 5. Schizothrix arenaria } \\
\text { (Berkeley) Gomont }\end{array}$ & S & $\mathrm{Hm}$ & I & $\mathrm{P}$ & & 74 & $\mathrm{Ee}$ \\
\hline $\begin{array}{l}\text { RHODOPHYCEAE } \\
\text { Goniotrichaceae } \\
\text { 6. Stylonema alsidii } \\
\text { (Zanardini) Drew }\end{array}$ & $S$ & O & I & $P$ & & 29 & $\mathrm{Ee}$ \\
\hline $\begin{array}{l}\text { Erythropeltidaceae } \\
\text { 7. Erythrocladia irregularis } \\
\text { Rosenvinge }\end{array}$ & $S$ & $\mathrm{O}$ & I & $\mathrm{P}$ & & $\mathrm{S} / \mathrm{Th}$ & $\mathrm{Ee}$ \\
\hline $\begin{array}{l}\text { Acrochaetiaceae } \\
\text { 8. Audouinella microscopica } \\
\text { (Nägeli ex Kützing) } \\
\text { Woelkerling }\end{array}$ & $S$ & $\mathrm{Mn}$ & I & $\mathrm{P}$ & & 11 & $\mathrm{Ee}$ \\
\hline $\begin{array}{l}\text { Helminthocladiaceae } \\
\begin{array}{l}\text { 9. Liagora ceranoides } \\
\text { Lamouroux }\end{array}\end{array}$ & $S$ & Gf & I & $\mathrm{P}$ & $\mathrm{R}$ & & 7906 \\
\hline \begin{tabular}{|l} 
Nemaliaceae \\
10. Trichogloea herveyi \\
W. Taylor
\end{tabular} & $S$ & Gf & I & $\mathrm{P}$ & $\mathrm{R}$ & & 7914 \\
\hline \begin{tabular}{|c} 
Galaxauraceae \\
11. Galaxaura rugosa \\
(Ellis et Solander) \\
Lamouroux
\end{tabular} & $\begin{array}{l}B \\
S\end{array}$ & Gf & I & $P$ & $\mathrm{R}$ & & $\begin{array}{l}11181 \\
11182 \\
11183\end{array}$ \\
\hline \begin{tabular}{|c|} 
Gelidiaceae \\
12. Pterocladia caerulescens \\
(Kützing) Santelices
\end{tabular} & $S$ & & I & $P$ & $\mathrm{R}$ & & 11211 \\
\hline \begin{tabular}{|l} 
Gelidiellaceae \\
13. Gelidiella acerosa \\
(Forsskal) Feldmann \\
et Hamel
\end{tabular} & $\begin{array}{l}B \\
S\end{array}$ & $\mathrm{Te}$ & I & $P$ & $\mathrm{R}$ & & $\begin{array}{c}E z \\
6705 \\
11212\end{array}$ \\
\hline
\end{tabular}


Cuadro 1. Continuación.

\begin{tabular}{|c|c|c|c|c|c|c|c|}
\hline Especies & Presencia & \begin{tabular}{|l|} 
Repro- \\
ducción \\
\end{tabular} & $\begin{array}{c}\text { Nivel } \\
\text { marea }\end{array}$ & $\begin{array}{l}\text { Exp. al } \\
\text { oleaje }\end{array}$ & Sustrato & $\begin{array}{l}\text { Epífita } \\
\text { de }\end{array}$ & $\begin{array}{l}\text { Observa- } \\
\text { ciones }\end{array}$ \\
\hline $\begin{array}{l}\text { Corallinaceae } \\
\text { 14. Amphiroa fragilissima } \\
\text { (Linnaeus) Lamouroux }\end{array}$ & $\begin{array}{l}B \\
S\end{array}$ & $\mathrm{Te}$ & 1 & $P$ & $\mathrm{R}$ & & 7923 \\
\hline $\begin{array}{l}\text { 15. A. rigida var. antillana } \\
\text { Boergesen }\end{array}$ & $\begin{array}{l}B \\
S\end{array}$ & $\mathrm{Te}$ & I & $P$ & $\mathrm{R}$ & & 7924 \\
\hline $\begin{array}{l}\text { 16. Hydrolithon farinosum } \\
\text { (Lamouroux) Penrose } \\
\text { et Chamberlain }\end{array}$ & $\begin{array}{l}B \\
S\end{array}$ & $\mathrm{Te}$ & I & $\mathrm{P}$ & & $\mathrm{S} / \mathrm{Th}$ & $\begin{array}{c}\text { Ee } \\
7912 \\
10260\end{array}$ \\
\hline $\begin{array}{l}\text { 17. H. improcerum (Foslie) } \\
\text { Foslie et Howe }\end{array}$ & $\begin{array}{l}B \\
S\end{array}$ & $\mathrm{Te}$ & I & $P$ & $\mathrm{R}$ & & 7930 \\
\hline $\begin{array}{l}\text { 18. Jania adhaerens } \\
\text { Lamouroux }\end{array}$ & S & & I & $P$ & & $\begin{array}{l}10 \\
11 \\
38\end{array}$ & $\begin{array}{c}E \\
7927\end{array}$ \\
\hline $\begin{array}{l}\text { 19. Lithophyllum bermudense } \\
\text { Foslie et Howe }\end{array}$ & $\begin{array}{l}B \\
S\end{array}$ & $\mathrm{Te}$ & I & $P$ & $\mathrm{R}$ & & $\begin{array}{l}7919 \\
7920\end{array}$ \\
\hline $\begin{array}{l}\text { 20. L. congestum (Foslie) } \\
\text { Foslie }\end{array}$ & S & $\mathrm{Te}$ & I & $P$ & $\mathrm{R}$ & & 7921 \\
\hline $\begin{array}{l}\text { 21. L. daedaleum Foslie et } \\
\text { Howe? }\end{array}$ & $S$ & $\mathrm{Te}$ & I & $P$ & $\mathrm{R}$ & & 7915 \\
\hline $\begin{array}{l}\text { 22. L. daedaleum var. pseu- } \\
\text { dodentatum Foslie et } \\
\text { Howe }\end{array}$ & $S$ & $\mathrm{Te}$ & 1 & $P$ & $\mathrm{R}$ & & 7916 \\
\hline 23. L. intermedium Foslie & $\begin{array}{l}B \\
S\end{array}$ & $\mathrm{Te}$ & 1 & $P$ & $\mathrm{R}$ & & $\begin{array}{l}7917 \\
7918 \\
7928\end{array}$ \\
\hline $\begin{array}{l}\text { 24. Neogoniolithon mamillare } \\
\text { (Harvey) Setchell et } \\
\text { Mason }\end{array}$ & S & $\mathrm{Te}$ & I & $P$ & $\mathrm{R}$ & & 7926 \\
\hline $\begin{array}{l}\text { 25. N. spectabile (Foslie) } \\
\text { Setchell et Mason }\end{array}$ & S & $\begin{array}{l}\text { Gf } \\
\mathrm{Te}\end{array}$ & I & $P$ & $\mathrm{R}$ & & 7929 \\
\hline $\begin{array}{l}\text { 26. Paragoniolithon solubile } \\
\text { (Foslie et Howe) Adey, } \\
\text { Townsend et Boykins }\end{array}$ & S & $\begin{array}{l}\text { Gf } \\
\mathrm{Te}\end{array}$ & I & $P$ & $\mathrm{R}$ & & 7933 \\
\hline
\end{tabular}


Acta Botánica Mexicana (1996), 36:59-75

Cuadro 1. Continuación.

\begin{tabular}{|c|c|c|c|c|c|c|c|}
\hline Especies & Presencia & $\begin{array}{l}\text { Repro- } \\
\text { ducción }\end{array}$ & $\begin{array}{c}\text { Nivel } \\
\text { marea }\end{array}$ & $\begin{array}{l}\text { Exp. al } \\
\text { oleaje }\end{array}$ & Sustrato & $\begin{array}{l}\text { Epífita } \\
\text { de }\end{array}$ & $\begin{array}{l}\text { Observa- } \\
\text { ciones }\end{array}$ \\
\hline $\begin{array}{l}\text { 27. Pneophyllum fragile } \\
\text { Kützing }\end{array}$ & $\begin{array}{l}B \\
S\end{array}$ & & I & $P$ & & $\mathrm{~S} / \mathrm{Th}$ & $\begin{array}{c}\mathrm{Ee} \\
7922\end{array}$ \\
\hline $\begin{array}{l}\text { Hypneaceae } \\
\text { 28. Hypnea spinella (C. } \\
\text { Agardh) Kützing }\end{array}$ & $S$ & & I & $P$ & $\mathrm{R}$ & & $\begin{array}{c}7905 \\
11203 \\
11204\end{array}$ \\
\hline $\begin{array}{l}\text { Gracilariaceae } \\
\text { 29. Gelidiopsis variabilis (J. } \\
\text { Agardh) Schmitz }\end{array}$ & $\begin{array}{l}B \\
S\end{array}$ & $\mathrm{Te}$ & I & $P$ & $\mathrm{R}$ & & $\begin{array}{c}6713 \\
11197\end{array}$ \\
\hline $\begin{array}{l}\text { Champiaceae } \\
\text { 30. Champia parvula (C. } \\
\text { Agardh) Harvey }\end{array}$ & $S$ & $\begin{array}{l}\text { Gf } \\
\mathrm{Te}\end{array}$ & I & $P$ & & $\mathrm{~S} / \mathrm{Th}$ & $\begin{array}{c}\mathrm{Ee} \\
6818 \\
11284\end{array}$ \\
\hline $\begin{array}{l}\text { Ceramiaceae } \\
\begin{array}{l}\text { 31. Centroceras clavulatum } \\
\text { (C. Agardh) Montagne }\end{array}\end{array}$ & $S$ & $\mathrm{Te}$ & I & $P$ & $\mathrm{R}$ & $\mathrm{S} / \mathrm{Th}$ & $\begin{array}{c}\mathrm{Ee} \\
10712\end{array}$ \\
\hline $\begin{array}{l}\text { 32. Ceramium flaccidum } \\
\text { (Kützing) Ardissone }\end{array}$ & $S$ & $\mathrm{Te}$ & I & $\mathrm{P}$ & $\mathrm{R}$ & $\mathrm{S} / \mathrm{Th}$ & $\begin{array}{c}\mathrm{Ee} \\
10699 \\
11198\end{array}$ \\
\hline $\begin{array}{l}\text { 33. Ceramium luetzelburgii } \\
\text { Schmidt }\end{array}$ & $S$ & $\mathrm{Te}$ & I & $P$ & $\mathrm{R}$ & & 8157 \\
\hline $\begin{array}{l}\text { 34. Ceramium nitens (C. } \\
\text { Agardh) J. Agardh }\end{array}$ & $\begin{array}{l}\mathrm{B} \\
\mathrm{S}\end{array}$ & & I & $P$ & $\mathrm{R}$ & 73 & $\begin{array}{c}\mathrm{Ee} \\
10709 \\
11199\end{array}$ \\
\hline $\begin{array}{l}\text { 35. Spyridia filamentosa } \\
\text { (Wulfen) Harvey }\end{array}$ & $S$ & & I & $P$ & $\mathrm{R}$ & $\mathrm{S} / \mathrm{Th}$ & $\begin{array}{c}\text { Ee } \\
11201\end{array}$ \\
\hline $\begin{array}{c}\text { Rhodomelaceae } \\
\text { 36. Acanthophora spicifera } \\
\text { (Vahl) Boergesen }\end{array}$ & $S$ & & I & $P$ & $\mathrm{R}$ & & $\begin{array}{c}7904 \\
11190\end{array}$ \\
\hline $\begin{array}{l}\text { 37. Chondria curvilineata } \\
\text { Collins et Hervey }\end{array}$ & $S$ & $\begin{array}{l}\text { Gf } \\
\mathrm{Te}\end{array}$ & I & $P$ & & $\mathrm{~S} / \mathrm{Th}$ & $\begin{array}{c}\mathrm{Ee} \\
11191\end{array}$ \\
\hline
\end{tabular}


Cuadro 1. Continuación.

\begin{tabular}{|c|c|c|c|c|c|c|c|}
\hline Especies & Presencia & $\begin{array}{l}\text { Repro- } \\
\text { ducción }\end{array}$ & $\begin{array}{l}\text { Nivel } \\
\text { marea }\end{array}$ & $\begin{array}{l}\text { Exp. al } \\
\text { oleaje }\end{array}$ & Sustrato & $\begin{array}{l}\text { Epífita } \\
\text { de }\end{array}$ & $\begin{array}{l}\text { Observa- } \\
\text { ciones }\end{array}$ \\
\hline $\begin{array}{l}\text { 38. Digenea simplex (Wulfen) } \\
\text { C. Agardh }\end{array}$ & $\begin{array}{l}B \\
S\end{array}$ & & I & $P$ & $\mathrm{R}$ & & $\begin{array}{c}7903 \\
11185 \\
11186\end{array}$ \\
\hline $\begin{array}{l}\text { 39. Laurencia obtusa (Hudson) } \\
\text { Lamouroux }\end{array}$ & S & & I & $P$ & $R$ & & 11188 \\
\hline $\begin{array}{c}\text { 40. Laurencia papillosa (C. } \\
\text { Agardh) Greville }\end{array}$ & $S$ & & I & $P$ & $R$ & & 11187 \\
\hline $\begin{array}{l}\text { 41. L. poiteaui (Lamouroux) } \\
\text { Howe }\end{array}$ & $S$ & $\begin{array}{l}\text { Gf } \\
\mathrm{Te}\end{array}$ & I & $P$ & $\mathrm{R}$ & & 11189 \\
\hline $\begin{array}{l}\text { 42. Polysiphonia sphaerocarpa } \\
\text { Boergesen }\end{array}$ & $S$ & $\begin{array}{l}\mathrm{Gm} \\
\mathrm{Gf} \\
\mathrm{Te}\end{array}$ & I & $P$ & & $\mathrm{~S} / \mathrm{Th}$ & $\begin{array}{l}7911 \\
\mathrm{Ee}\end{array}$ \\
\hline $\begin{array}{l}\text { BACILLARIOPHYCEAE } \\
\text { Discaceae } \\
\text { 43. Melosira borreri Greville }\end{array}$ & $\begin{array}{l}B \\
S\end{array}$ & & I & $P$ & $R$ & $\begin{array}{l}10 \\
11 \\
52\end{array}$ & E \\
\hline $\begin{array}{l}\text { 44. Melosira moniliformis } \\
\text { (Müller) C. Agardh }\end{array}$ & $S$ & & 1 & $P$ & & $\begin{array}{l}10 \\
30 \\
79\end{array}$ & E \\
\hline $\begin{array}{c}\text { Biddulphiaceae } \\
\text { 45. Biddulphia pulchella Gray }\end{array}$ & $\begin{array}{l}B \\
S\end{array}$ & & I & $P$ & & $\begin{array}{l}10 \\
11 \\
52\end{array}$ & $E$ \\
\hline $\begin{array}{l}\text { Fragilariaceae } \\
\text { 46. Grammathophora marina } \\
\text { (Lyngbye) Kützing }\end{array}$ & $S$ & & I & $P$ & & $\begin{array}{ll}10 & 33 \\
29 & 52 \\
30 & 79\end{array}$ & E \\
\hline $\begin{array}{l}\text { 47. Licmophora abbreviata } \\
\text { C. Agardh }\end{array}$ & $S$ & & I & $P$ & & $\begin{array}{l}10 \\
29 \\
52\end{array}$ & $E$ \\
\hline $\begin{array}{l}\text { 48. Licmophora flabellata } \\
\text { (Greville) C. Agardh }\end{array}$ & S & & I & $P$ & & $\begin{array}{l}10 \\
30 \\
52\end{array}$ & E \\
\hline
\end{tabular}


Acta Botánica Mexicana (1996), 36:59-75

Cuadro 1. Continuación.

\begin{tabular}{|c|c|c|c|c|c|c|c|}
\hline Especies & Presencia & $\begin{array}{l}\text { Repro- } \\
\text { ducción }\end{array}$ & $\begin{array}{l}\text { Nivel } \\
\text { marea }\end{array}$ & $\begin{array}{l}\text { Exp. al } \\
\text { oleaje }\end{array}$ & Sustrato & $\begin{array}{l}\text { Epífita } \\
\text { de }\end{array}$ & $\begin{array}{l}\text { Observa- } \\
\text { ciones }\end{array}$ \\
\hline $\begin{array}{l}\text { Achnantaceae } \\
\text { 49. Achnantes longipes } \\
\text { C. Agardh }\end{array}$ & $S$ & & I & $P$ & & $\begin{array}{l}10 \\
33 \\
52\end{array}$ & $E$ \\
\hline $\begin{array}{l}\text { 50. Cocconeis placentula } \\
\text { Ehrenberg }\end{array}$ & $\begin{array}{l}B \\
S\end{array}$ & & I & $\mathrm{P}$ & & $\begin{array}{ll}10 & 33 \\
11 & 52 \\
29 & 78 \\
30 & \end{array}$ & $E$ \\
\hline $\begin{array}{l}\text { PHAEOPHYCEAE } \\
\text { Chordariaceae } \\
\text { 51. Cladosiphon zosterae } \\
\text { (J. Agardh) Kylin }\end{array}$ & S & $U$ & 1 & $P$ & & $\mathrm{~S} / \mathrm{Th}$ & $\begin{array}{c}\mathrm{Ee} \\
7913\end{array}$ \\
\hline $\begin{array}{c}\text { Sphacelariaceae } \\
\text { 52. Sphacelaria tribuloides } \\
\text { Meneghini }\end{array}$ & $S$ & $\mathrm{Pp}$ & $\mathrm{L}$ & Ex & $\mathrm{R}$ & & $\begin{array}{c}9737 \\
11208\end{array}$ \\
\hline $\begin{array}{l}\text { Dictyotaceae } \\
\text { 53. Dictyota bartayresiana } \\
\text { Lamouroux }\end{array}$ & $\begin{array}{l}B \\
S\end{array}$ & Gf & I & $P$ & $\mathrm{R}$ & $\mathrm{S} / \mathrm{Th}$ & $\begin{array}{c}\mathrm{Ee} \\
11171 \\
11172 \\
10625\end{array}$ \\
\hline $\begin{array}{l}\text { 54. D. bartayresiana var. } \\
\text { divaricata (J. Agardh) } \\
\text { J. Agardh }\end{array}$ & $S$ & Gf & 1 & $\mathrm{P}$ & & $\mathrm{S} / \mathrm{Th}$ & $\begin{array}{c}11173 \\
\mathrm{Ee}\end{array}$ \\
\hline 55. D. cervicornis Kützing & $S$ & & I & $P$ & $\mathrm{R}$ & & 11175 \\
\hline $\begin{array}{l}\text { 56. Padina boergesenii } \\
\text { Allender et Kraft }\end{array}$ & $\begin{array}{l}B \\
S\end{array}$ & Gf & I & $\mathrm{P}$ & $\mathrm{R}$ & & $\begin{array}{l}11176 \\
11177 \\
11178\end{array}$ \\
\hline $\begin{array}{c}\text { CHLOROPHYCEAE } \\
\text { Ulvaceae } \\
\text { 57. Enteromorpha compressa } \\
\text { (Linnaeus) Nees }\end{array}$ & $S$ & & $\mathrm{~L}$ & $\begin{array}{c}\text { Ex } \\
1\end{array}$ & $\begin{array}{l}R \\
P\end{array}$ & & 10694 \\
\hline
\end{tabular}


Cuadro 1. Continuación.

\begin{tabular}{|c|c|c|c|c|c|c|c|}
\hline Especies & Presencia & \begin{tabular}{|l|} 
Repro- \\
ducción \\
\end{tabular} & $\begin{array}{c}\text { Nivel } \\
\text { marea }\end{array}$ & $\begin{array}{l}\text { Exp. al } \\
\text { oleaje }\end{array}$ & Sustrato & $\begin{array}{c}\text { Epífita } \\
\text { de }\end{array}$ & $\begin{array}{c}\text { Observa- } \\
\text { ciones }\end{array}$ \\
\hline $\begin{array}{l}\text { Siphonocladaceae } \\
\text { 58. Boodlea composita } \\
\text { (Harvey) Brand }\end{array}$ & S & Sc & L & Ex & $\mathrm{R}$ & & 11207 \\
\hline $\begin{array}{l}\text { 59. Cladophoropsis macro- } \\
\text { meres W. Taylor }\end{array}$ & $\begin{array}{l}B \\
S\end{array}$ & Sc & 1 & $P$ & $\mathrm{R}$ & & 11206 \\
\hline $\begin{array}{l}\text { 60. C. membranacea (C. } \\
\text { Agardh) Boergesen }\end{array}$ & $S$ & & 1 & $P$ & $\mathrm{R}$ & & 11205 \\
\hline $\begin{array}{l}\text { 61. Struvea anastomosans } \\
\text { (Harvey) Piccone et } \\
\text { Grunow }\end{array}$ & S & & I & $P$ & $\mathrm{R}$ & & 7907 \\
\hline $\begin{array}{c}\text { Valoniaceae } \\
\text { 62. Dictyosphaeria cavernosa } \\
\text { (Forsskal) Boergesen }\end{array}$ & $S$ & & L & Ex & $\mathrm{R}$ & & $\begin{array}{c}8941 \\
11213\end{array}$ \\
\hline $\begin{array}{l}\text { 63. Ernodesmis verticillata } \\
\text { (Kützing) Boergesen }\end{array}$ & $S$ & Sc & । & $P$ & $\mathrm{R}$ & & 8449 \\
\hline $\begin{array}{l}\text { Anadyomenaceae } \\
\text { 64. Anadyomene stellata } \\
\text { (Wulfen) C. Agardh }\end{array}$ & $\begin{array}{l}B \\
S\end{array}$ & & L & Ex & $\mathrm{R}$ & & 11202 \\
\hline $\begin{array}{l}\text { Cladophoraceae } \\
\text { 65. Cladophora coelothrix } \\
\text { Kützing }\end{array}$ & $S$ & & I & $P$ & $\mathrm{R}$ & & 10656 \\
\hline 66. C. montagneana Kützing & S & & I & $P$ & $\mathrm{R}$ & & 7909 \\
\hline 67. C. socialis Kützing & $S$ & & I & $P$ & $\mathrm{R}$ & & $\begin{array}{l}11209 \\
11210\end{array}$ \\
\hline $\begin{array}{l}\text { Bryopsidaceae } \\
\text { 68. Bryopsis hypnoides } \\
\text { Lamouroux }\end{array}$ & $S$ & & I & $P$ & $\mathrm{R}$ & & 7936 \\
\hline 69. B. pennata Lamouroux & S & & L & Ex & $\mathrm{R}$ & & 7937 \\
\hline
\end{tabular}


Acta Botánica Mexicana (1996), 36:59-75

Cuadro 1. Continuación.

\begin{tabular}{|c|c|c|c|c|c|c|c|}
\hline Especies & Presencia & $\begin{array}{l}\text { Repro- } \\
\text { ducción }\end{array}$ & $\begin{array}{l}\text { Nivel } \\
\text { marea }\end{array}$ & $\begin{array}{l}\text { Exp. al } \\
\text { oleaje }\end{array}$ & Sustrato & $\begin{array}{c}\text { Epífita } \\
\text { de }\end{array}$ & $\begin{array}{l}\text { Observa- } \\
\text { ciones }\end{array}$ \\
\hline Codiaceae & & & & & & & \\
\hline 70. Codium taylorii Silva & $S$ & G & I & $\mathrm{P}$ & $\mathrm{R}$ & & 6794 \\
\hline Caulerpaceae & & & & & & & \\
\hline $\begin{array}{l}\text { 71. Caulerpa cupressoides } \\
\text { (Vahl) C. Agardh }\end{array}$ & $\begin{array}{l}\mathrm{B} \\
\mathrm{C}\end{array}$ & & I & $\mathrm{P}$ & A & & 11180 \\
\hline $\begin{array}{l}\text { 72. C. racemosa (Forsskäl) } \\
\text { J. Agardh }\end{array}$ & $\begin{array}{l}B \\
S\end{array}$ & & I & $\mathrm{P}$ & A & & 7246 \\
\hline $\begin{array}{l}\text { 73. C. sertularioides (S. } \\
\text { Gmelin) Howe }\end{array}$ & $\begin{array}{l}B \\
S\end{array}$ & & I & $P$ & A & & 11179 \\
\hline $\begin{array}{l}\text { Udoteaceae } \\
\text { 74. Halimeda opuntia } \\
\text { (Linnaeus) Lamouroux }\end{array}$ & $\begin{array}{l}B \\
S\end{array}$ & & I & $P$ & A & & $\begin{array}{c}5968 \\
11194 \\
10583\end{array}$ \\
\hline $\begin{array}{l}\text { 75. H. tuna (Ellis et Solander) } \\
\text { Lamouroux }\end{array}$ & $\begin{array}{l}B \\
S\end{array}$ & & I & $P$ & A & & $\begin{array}{l}11192 \\
11193\end{array}$ \\
\hline $\begin{array}{l}\text { 76. Rhipocephalus phoenix f. } \\
\text { longifolius A. et E. S. } \\
\text { Gepp }\end{array}$ & $\begin{array}{l}B \\
S\end{array}$ & & I & $P$ & A & & $\begin{array}{c}6072 \\
11195 \\
11196\end{array}$ \\
\hline $\begin{array}{l}\text { Dasycladaceae } \\
\text { 77. Cymopolia barbata } \\
\text { (Linnaeus) Lamouroux }\end{array}$ & B & $G$ & 1 & $P$ & $\mathrm{R}$ & & 1602 \\
\hline 78. Neomeris annulata Dickie & $\begin{array}{l}B \\
S\end{array}$ & G & I & $P$ & $\mathrm{R}$ & & \\
\hline $\begin{array}{l}\text { Polyphysaceae } \\
\text { 79. Acetabularia schenckii } \\
\text { Möbius }\end{array}$ & $S$ & $G$ & I & $P$ & $\mathrm{R}$ & & 10135 \\
\hline $\begin{array}{l}\text { 80. Polyphysa polyphysoides } \\
\text { (Crouan frat.) Schnetter }\end{array}$ & $S$ & $G$ & 1 & $P$ & $\mathrm{R}$ & & \\
\hline
\end{tabular}




\section{SIMBOLOGIA}

\section{Presencia}

B - Estación climática de lluvias (mayo, 1983)

S - Estación climática de secas (febrero, diciembre de 1984; noviembre de 1985, 1986 y 1992)

$\begin{array}{lccc}\text { Reproducción } & \text { Nivel de marea } & \begin{array}{c}\text { Exposición al } \\ \text { oleaje }\end{array} & \text { Sustrato } \\ \text { O - Monosporas } & \text { L - Intermareal } & \text { Ex - Expuesto } & \text { R - Rocas } \\ \text { Te - Tetrasporas } & \text { I - Submareal por buceo } & \text { P - Protegido } & \text { A - Arenoso } \\ \text { Gm - Gametos masculinos } & \text { libre }(0.5-2.0 \mathrm{~m}) & \\ \text { Gf - Gametos femeninos } & & \\ \text { Pp - Propágulos } & & \\ \text { U - Unangias uniloculares } & & \\ \text { G - Gametangios } & & \\ \text { Sc - Segregación celular } & \\ \text { Ht - Heterocistos } & \\ \text { Hm - Hormogonios } & \\ \text { La ausencia del símbolo co- } & \\ \text { rresponde al estado vegetativo } & \end{array}$

Epífita de: Los números que se indican en esta columna corresponden a las algas sobre las que se localizaron las especies epífitas.

Las especies epífitas a menudo se encuentran herborizadas con su hospedero, por lo tanto corresponden al mismo número de registro de éste.

Observaciones: E - Epífita; Ee - Epífita estricta; S/Th - Epífita de Thalassia testudinum; Ez - Epizoica. Los números que se localizan en esta columna corresponden al registro del herbario ENCB.

\section{LITERATURA CITADA}

Anagnostidis, K., y J. Komárek. 1988. Modern approach to the classification system of cyanophytes. 3. Oscillatoriales. Archiv fur Hydrobiologie, Supplement 80: 327-472.

Anónimo, 1982. Comportamiento fisicoquímico del agua en el arrecife de Isla Verde, Ver. Dirección General de Oceanografría, México, D.F. 20 pp.

Chávez, E. A. 1973. Observaciones generales sobre las comunidades del Arrecife Lobos, Veracruz. An. Esc. Nac. Cienc. Biol. Méx. 20: 13-21.

Dawes, C. I. 1986. Botánica marina. Ed. Limusa México, D.F. 673 pp.

de la Lanza, E. G. 1991. Oceanografía de mares mexicanos. Ed. A. G. T. México, D.F. 569 pp.

Díaz-Piferrer, M. 1967. Las algas superiores y fanerógamas marinas. In: Ecología Marina. Fundación La Salle. Caracas. pp. 273-307.

Emery, K. O. 1963. Coral reefs off Veracruz, México. Geofís. Int. 3: 11-17.

Fritsch, F. E. 1935. Structure and reproduction of the algae. Cambridge University Press. Cambridge. Vol. 1. $791 \mathrm{pp}$. 
García, E. 1973. Modificaciones al sistema de clasificación climática de Koeppen (Adaptada para la República Mexicana). Instituto de Geografía, Universidad Nacional Autónoma de México. México D.F. 246 pp.

Goff, L. J. y A. W. Coleman. 1985. The role of secondary pit connections in red algal parasitism. J. Phycol. 21: 483-508.

Henkel, D. H. 1982. Echinoderms of Enmedio Reef, Southwestern Gulf of Mexico. Tesis. Corpus Christi State Univ. Corpus Christi, Texas. 78 pp.

Huerta M., L. 1960. Lista preliminar de las algas marinas del litoral del estado de Veracruz, México. Bol. Soc. Bot. México 25: 39-45.

Huerta M., L. y M. A. Garza Barrientos. 1964. Algas marinas de la Barra de Tuxpan y los arrecifes Blanquilla y Lobos. An. Esc. Nac. Cienc. Biol. Méx. 13(1-4): 5-21.

Huerta-Múzquiz, L., M. E. Sánchez-Rodríguez y M. L. Chávez-Barrera. 1977. Algas marinas de Isla de Enmedio, Veracruz. In: Mem. V. Congr. Nac. Oceanogr. México, D.F. pp. 314-325.

Huisman, J. M., y M. A. Borowitza. 1990. A revision of the Australian species of Galaxaura (Rhodophyta, Galaxauraceae), with a description of Tricleocarpa gen. nov. Phycologia 29: 150-172.

Humm, H. J. y H. Hildenbrand. 1962. Marine algae from the Gulf coast of Texas and Mexico. Publs. Inst. Mar. Sci. Univ. Tex. 8: 227-268.

Humm, H. J. y S. R. Wicks. 1980. Introduction and guide to the marine bluegreen algae. Wiley. Nueva York. 194 pp.

Ibarra-Obando, S. E. y R. Aguilar-Rosas. 1985. Macroalgas flotantes y epífitas asociadas con Zostera marina en Bahía San Quintin (B.C., México), durante verano-otoño 1982. Biomasa y composición taxonómica. Ciencias Marinas 11(3): 89-104.

Komárek, J. y K. Anagnostidis. 1986. Modern approach to the clasification system of Cyanophyta. 2. Chroococcales. Arch. Hydrobiol. Supplement 73(2): 157-226.

Lehman, R. L. y J. W. Tunell. 1992. Species composition and ecology of the macroalgae of Enmedio Reef, Veracruz, Mexico. Texas J. Scien. 44(4): 445-457.

Littler, M. M., D. S. Littler y P. R. Taylor. 1983. Evolutionary strategies in a tropical barrier reef system: functional-form groups of marine macroalgae. J. Phycol. 19: 229-237.

Lot-Helgueras, A. 1968. Estudios sobre fanerógamas marinas en las cercanías de Veracruz, Ver. Tesis de Licenciatura. Facultad de Ciencias, Universidad Nacional Autónoma de México. México, D.F. $66 \mathrm{pp}$.

Lot-Helgueras, A. 1971. Estudios sobre fanerógamas marinas en las cercanías de Veracruz. Veracruz. An. Inst. Biol. Universidad Nacional Autónoma de México. Ser. Bot. 1: 1-48.

Lüning, K. 1990. Seaweeds, their enviroment, biogeography and ecophysiology. Willey Interscience. Nueva York. 527 pp.

Mendoza-González., A. C. y L. E. Mateo-Cid. 1985. Contribución al conocimiento de la flora marina bentónica de las islas Sacrificios y Santiaguillo, Veracruz. México. Phytologia 59(1): 9-16.

Norris, R. E. 1987. The systematic position of Gelidiopsis and Ceratodyction (Gigartinales, Rhodophyceae), genera new to South Africa. South African Journal of Botany 53: 495-506.

Quintana y Molina, J. 1991. Resultados del programa de investigaciones en arrecifes veracruzanos del Laboratorio de Sistemas Bentónicos Litorales. Hidrobiológica 1(1): 73-86.

Penrose, D. y W. J. Woelkerling. 1991. Pneophyllum fragile in southern Australia: implications for generic concepts in the Masthophoroideae (Corallinaceae, Rhodophyta). Phycologia 30: 495-506.

Penrose, D. y Y. Chamberlain. 1993. Hydrolithon farinosum (Lamouroux) comb. nov.: implications for generic concepts in the Mastophoroideae (Corallinaceae, Rhodophyta). Phycologia 32(4): 295-303.

Rigby, J. K. y W. G. McIntire. 1966. Isla de Lobos and associated reefs, Veracruz, México. Brigham Young Univ. Geol. Stud. 13: 1-46.

Round, F. E. 1981. The ecology of algae. Cambridge University Press. Nueva York. 653 pp.

Santelices, B. 1977. Ecología de las algas marinas bentónicas. Univ. Católica de Chile. Santiago. 384 pp. 
Schneider, C. W y R. B. Searles. 1991. Seaweeds of the Southeastern United States. Duke University Press. Durham. 553 pp.

Taylor, W. R. 1960. Marine algae of the eastern tropical and subtropical coast of the Americas. Univ. Mich. Press. Ann Arbor. 870 pp.

Tunnell, J. W. 1988. Regional comparison of southwestern Gulf of Mexico to Caribbean Sea coral reef. In: Proceed. $6^{\text {th }}$ Internat. Coral Reef Symp. Sydney. pp. 303-308.

Vargas-Hernández, J. M., A. Hernández-Gutiérrez y L. F. Carrera-Parra. 1993. Sistema arrecifal veracruzano. In: Biodiversidad marina y costera de México. Comisión Nacional para el Conocimiento y Uso de la Biodiversidad. México, D.F. pp. 559-575.

Villalobos, A. 1971. Estudios ecológicos en un arrecife coralino en Veracruz, México. In: Symp. Invest. Resour. Caribbean Sea. UNESCO y FAO pp. 532-545.

Werner, D. (ed.). 1977. The biology of diatoms. Blackwell Scientific Publications. Oxford. 498 pp.

Wynne, M. J. 1986. A checklist of benthic marine algae of the tropical and subtropical western Atlantic. Can. J. Bot. 64: 2239-2281. 